\title{
Nonintegrable discrete-time driftless control systems: geometric phases beyond the area rule
}

\author{
Claudio Altafini \\ Book Chapter \\ Tweet
}

N.B.: When citing this work, cite the original article.

Part of: 2016 IEEE 55th Conference on Decision and Control (CDC), 2016, pp. 4692-4697. ISBN: 9781509018376,9781509018444 and 9781509018383

DOI: http://dx.doi.org/10.1109/CDC.2016.7798984

Copyright: IEEE Press

Available at: Linköping University Electronic Press http://urn.kb.se/resolve?urn=urn:nbn:se:liu:diva-135542

LINKÖPINGS

UNIVERSITET 


\title{
Nonintegrable discrete-time driftless control systems: geometric phases beyond the area rule
}

\author{
C. Altafini \\ Division of Automatic Control, Dept. of Electrical Engineering, \\ Linköping University, SE-58183, Linköping, Sweden. \\ email: claudio.altafini@liu.se
}

\begin{abstract}
In a continuous-time nonlinear driftless control system, a geometric phase is a consequence of nonintegrability of the vector fields, and it describes how cyclic trajectories in shape space induce non-periodic motion in phase space, according to an area rule. The aim of this paper is to shown that geometric phases exist also for discrete-time driftless nonlinear control systems, but that unlike their continuous-time counterpart, they need not obey any area rule, i.e., even zeroarea cycles in shape space can lead to nontrivial geometric phases. When the discrete-time system is obtained through Euler discretization of a continuous-time system, it is shown that the zero-area geometric phase corresponds to the gap between the Euler discretization and an exact discretization of the continuous-time system.
\end{abstract}

\section{INTRODUCTION}

For nonlinear control systems, it is well known that nonintegrability conditions on the vector fields are at the basis of our notions of (nonlinear) controllability and observability [4], [20], as well as of many motion planning algorithms [12], [20]. If the system is driftless, then nonintegrability of the vector fields (and Lie bracket conditions) allows to produce motion in directions not spanned by the vector fields, as in the parallel parking of a car [10], [11], [19]. Derived motions like the "lateral" displacement of a car in a parallel parking are sometimes referred to as geometric phases, because they appear in systems in which a cyclic change in some of the variables (called shape variables) induces a non-zero net motion on other variables (called phase variables).

Geometric phases are normally studied in continuoustime [13], [12], [20] and in different fields, like classical mechanics [13], quantum mechanics [2], [22], molecular systems, [14], robotics [12], [20] and control theory [4]. When a continuous-time driftless nonlinear control system is nonintegrable, periodic inputs can be used to induce nonperiodic movements in the phase variable, see for instance [3], [7], [22] for applications to swimming bodies in fluids, [18] for the falling cat problem, and [10], [11], [19] for the already mentioned parallel parking of a car. The amplitude of the phase displacement is proportional to the area of the cyclic path in shape space. In particular, a zero-area cycle yields no geometric phase.

The aim of this paper is to shown that the situation is different for a discrete-time driftless nonlinear system, in the sense that even a zero-area cycle can induce a nontrivial geometric phase. Although a large body of literature exists on determining discrete-time equivalents of the nonlinear notions used in control theory [1], [8], [15], [16], [17], in our knowledge, the properties of discrete time geometric phases have never been investigated, let alone the existence of phase motions induced by zero-area shape cycles.

Such a geometric phase appears to be both path-dependent and sampling length dependent. In particular, it tends to zero when the sampling interval tends to zero. It is shown in the paper that when the discrete-time system is obtained through an approximate discretization of a nonlinear system using an Euler method, then the geometric phase is related to the truncation error with respect to an exact discretization obtained through a complete Taylor series expansion method.

\section{A MOtIVATING EXAMPLE: BROCKETT INTEGRATOR}

In this Section we consider a well-known case study, the Brockett nonholonomic integrator [5].

\section{A. Continuous-time case}

In continuous-time, the Brockett integrator is the driftless control system in $\mathbb{R}^{3}$

$$
\begin{aligned}
& \dot{x}_{1}=u_{1} \\
& \dot{x}_{2}=u_{2} \\
& \dot{x}_{3}=x_{1} u_{2} .
\end{aligned}
$$

In the following $x_{1}$ and $x_{2}$ will be denoted shape variables, and $x_{3}$ as phase variable. Assume that the control inputs $u_{1}$ and $u_{2}$ correspond to the piecewise constant trajectories shown in the top plot of panel (a) of Fig. 1. Then a cyclic motion is produced in shape space $\mathcal{S}$ which results in a net displacement in the phase variable $x_{3}$, see panel (b) of Fig. 1. The geometric interpretation of this result is that if we consider $x \in \mathcal{M} \subset \mathbb{R}^{3}$ and the projection to the shape space

$$
\begin{aligned}
\pi: \mathcal{M} & \rightarrow \mathcal{S} \subset \mathbb{R}^{2} \\
x & \mapsto x_{s}=\left[\begin{array}{l}
x_{1} \\
x_{2}
\end{array}\right]
\end{aligned}
$$

then, given $x(0)$, for each trajectory $\gamma:[0, t] \rightarrow \mathcal{S} \exists$ a unique $x(t) \in \mathcal{M}$ such that for the solution of (1), $x(t)=\pi^{-1}\left(\pi(x(t))\right.$, i.e., the geometric phase variable $x_{3}(t)$ corresponding to $\gamma(t)$ is unique. In particular, if $\Gamma:[0, T] \rightarrow$ 
$\mathcal{S}$ is a closed shape curve enclosing an area $\Omega$, then the geometric phase (or "holonomy", [4]) of $\Gamma$ is

$$
x_{3}(T)=x_{3}(0)+\oint_{\Gamma} x_{1} d x_{2}
$$

or, by Stokes theorem,

$$
\begin{aligned}
x_{3}(T) & =x_{3}(0)+\int_{\Omega} d\left(x_{1} d x_{2}\right) \\
& =x_{3}(0)+\int_{\Omega} d x_{1} d x_{2}=x_{3}(0)+\omega
\end{aligned}
$$

where $\omega$ is the area of $\Omega$.

When instead the input trajectories $u_{1}$ and $u_{2}$ are identical, so are those of the shape variables $x_{1}$ and $x_{2}$, meaning that a zero-area cyclic trajectory is produced in shape space, see panels (c) and (d) of Fig. 1. From (2), in this case the phase variable $x_{3}$ shows no net displacement at the end of the cycle, see also below for an alternative calculation.

\section{B. Discrete-time case}

Let us now consider a discretized version of the system (1), for instance obtained replacing the derivative operator with an Euler difference, with sampling time $h=\Delta t$ assumed constant:

$$
x(k+1)=x(k)+h\left[\begin{array}{l}
1 \\
0 \\
0
\end{array}\right] u_{1}(k)+h\left[\begin{array}{c}
0 \\
1 \\
x_{1}(k)
\end{array}\right] u_{2}(k) .
$$

For this system, the shape and phase variables corresponding to the same input patterns as in Fig. 1 are shown in Fig. 2. While the case of non-zero area shape cycle is similar (panels (a) and (b)), the case of zero-area shape cycle (panels (c) and (d)) is not. In particular a non-zero net displacement in the phase variable $x_{3}$ happens also when the area of the space cyclic trajectory is zero, see panels (c) and (d) of Fig. 2. If $\alpha$ is the modulus of the amplitude of the inputs $u_{1}$ and $u_{2}$ in these cyclic trajectories, then in the zero-area case the (identical) input profiles $u_{i}(i=1,2)$ are

$$
u_{i}(k)= \begin{cases}0 & k<k_{1} \\ \alpha & k_{1} \leqslant k<k_{2} \\ 0 & k_{2} \leqslant k<k_{3} \\ -\alpha & k_{3} \leqslant k<k_{4} \\ 0 & k \geqslant k_{4}\end{cases}
$$

where $k_{1}$ and $k_{2}$ are begin and end of the positive input step, $k_{3}$ and $k_{4}$ are begin and end of the negative input step $\left(k_{i}<k_{j}\right.$ whenever $i<j$ ). Denote $\ell=k_{2}-k_{1}=k_{4}-k_{3}$ the step length in number of samples, $\ell \geqslant 1(\ell=5$ in Fig. 2$)$. In correspondence of the input pattern (4), the solution of (3) is

- for $k \leqslant k_{1}$

$$
x(k)=x(0)
$$

- for $k_{1}<k \leqslant k_{2}$

$$
x(k)=x(0)+\left(k-k_{1}\right)\left[\begin{array}{c}
1 \\
1 \\
x_{2}(0)
\end{array}\right] h \alpha+\left[\begin{array}{c}
0 \\
0 \\
\sum_{i=1}^{k-k_{1}-1} i
\end{array}\right] h^{2} \alpha^{2}
$$

- for $k_{2}<k \leqslant k_{3}$

$$
x(k)=x(0)+\ell\left[\begin{array}{c}
1 \\
1 \\
x_{2}(0)
\end{array}\right] h \alpha+\left[\begin{array}{c}
0 \\
0 \\
\sum_{i=1}^{\ell-1} i
\end{array}\right] h^{2} \alpha^{2}
$$

- for $k_{3}<k \leqslant k_{4}$

$x(k)=x(0)+\left(k_{4}-k\right)\left[\begin{array}{c}1 \\ 1 \\ x_{2}(0)\end{array}\right] h \alpha+\left[\begin{array}{c}0 \\ 0 \\ \sum_{i=1}^{k_{4}-k} i-\ell\end{array}\right] h^{2} \alpha^{2}$

- for $k>k_{4}$

$$
x(k)=x(0)-\left[\begin{array}{l}
0 \\
0 \\
\ell
\end{array}\right] h^{2} \alpha^{2} .
$$

Since $x_{1}(k)=x_{2}(k) \forall k$, the shape trajectory has indeed zero area. Furthermore, since for $k>k_{4} x_{i}(k)=x_{i}(0)$, $i=1,2$, the shape trajectory is also periodic. However, for $k>k_{4}, x_{3}(k) \neq x_{3}(0)$, as a geometric phase proportional to $\ell h^{2} \alpha^{2}$ has been generated. Therefore it follows that an area rule like (2) cannot hold for discrete-time systems.

To see how such a geometric phase appears, let us look at the summations in (5)-(9): during the positive input step $\left(k_{1} \leqslant k<k_{2}\right)$, the $x_{3}$ variable builds up the partial sum $\sum_{i=1}^{\ell-1} i$ (starting from 0 , then 1 , until $\ell-1$ ). However, when entering the negative input step $\left(k_{3} \leqslant k<k_{4}\right)$, the first term subtracted to that summation is $\ell$ (then $\ell-1, \ell-2, \ldots, 1$, until complete erasure of the summation). It is this negative term $\ell$ which leads to a nonzero phase motion.

If the continuous-time input steps of Fig. 1 have amplitude 1 and area 1 , i.e., $\int_{t_{1}}^{t_{2}} u_{i}(\tau) d \tau=1$ where $t_{i}=k_{i} h$, then for the discrete-time system, with $\alpha=1$,

$$
\sum_{k=k_{1}}^{k_{2}} h u_{i}(k)=h\left(k_{2}-k_{1}\right)=h \ell .
$$

Hence when the sampling time $h \rightarrow 0$, the constraint $h \ell=1$ becomes $\lim _{h \rightarrow 0} h \ell=1$ i.e., $\lim _{h \rightarrow 0} \ell=\infty$ but $\lim _{h \rightarrow 0} \ell h^{2}=0$, meaning that in (9) the geometric phase disappears when the Euler difference converges to a continuous-time differential operator.

Apart from the sampling time, this zero-area geometric phase appears to be dependent also on the path followed. For instance, if we apply sinusoidal inputs $\left(u_{1}=u_{2}\right)$ to the system (3) then we obtain the phase shown in Fig. 3. When the sampling time is divided by two, the geometric phase accumulated along the sinusoidal path decreases. Also reducing the input amplitude (but not $h$ ) reduced the geometric phase.

\section{Geometric phase and exact discretization for the Brockett integrator}

If we use homogeneous coordinates $\bar{x}=\left[\frac{x}{1}\right]$, then (1) can be rewritten as the bilinear system

$$
\dot{\bar{x}}=\left(A_{1} u_{1}+A_{2} u_{2}\right) \bar{x}
$$



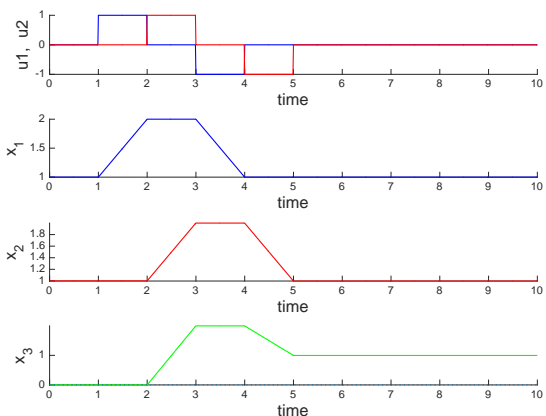

(a)
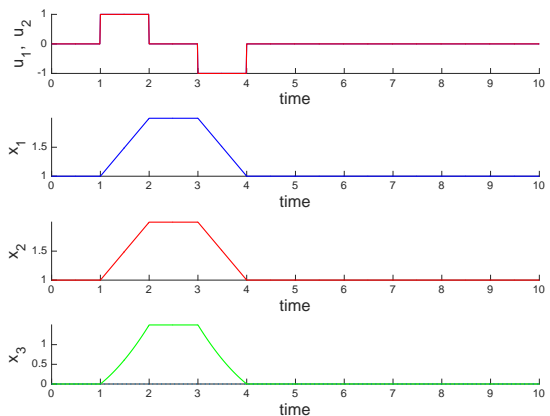

(c)

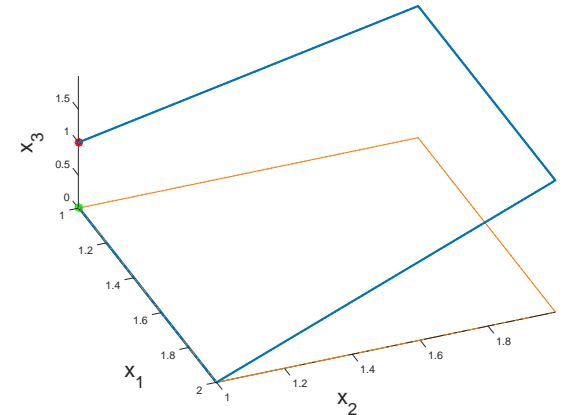

(b)

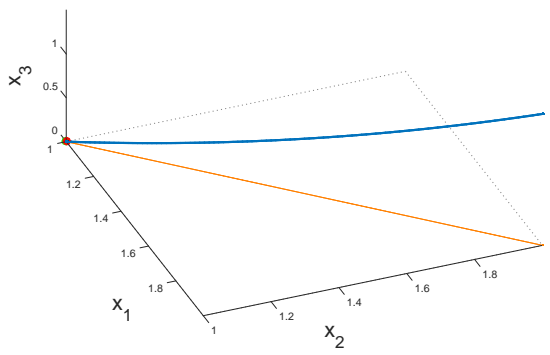

(d)

Fig. 1. Effect of cyclic shape trajectories in the continuous-time system (1). Left column: time profiles of the variables. Right column: corresponding shape (orange) and phase space (blue) profiles. The starting point is given in green and the end point in red. In the top row the area of the shape cycle in $\left(x_{1}, x_{2}\right)$ is nonzero, and so is the phase $\left(x_{3}\right)$ displacement. In the bottom row the shape cycle has zero area and so does the phase displacement.
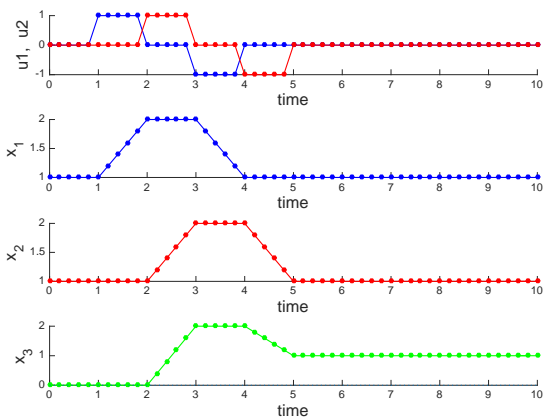

(a)
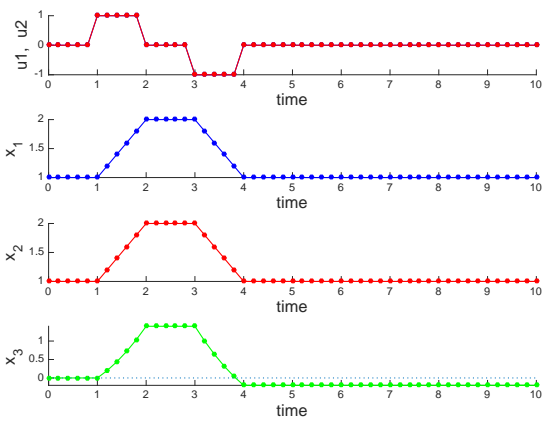

(c)

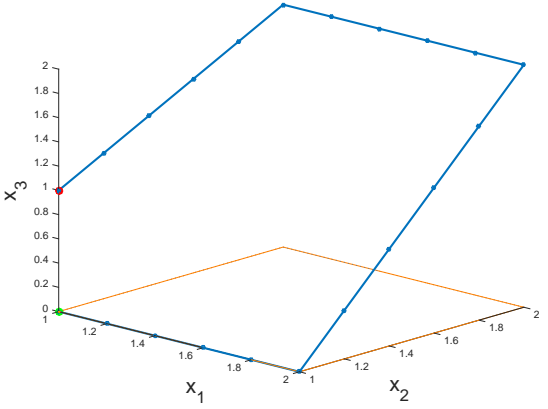

(b)

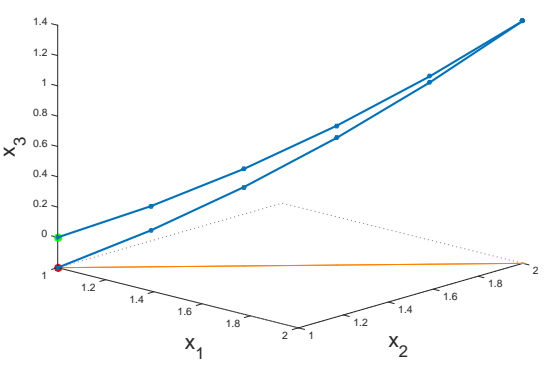

(d)

Fig. 2. Effect of cyclic shape trajectories in the discrete-time system (3). Left column: time profiles of the variables. Right column: corresponding shape (orange) and phase space (blue) profiles. The starting point is given in green and the end point in red. In the top row the area of the shape cycle in $\left(x_{1}, x_{2}\right)$ is nonzero, and so is the phase $\left(x_{3}\right)$ displacement. In the bottom row the shape cycle has zero area but the phase displacement is still nonzero. 

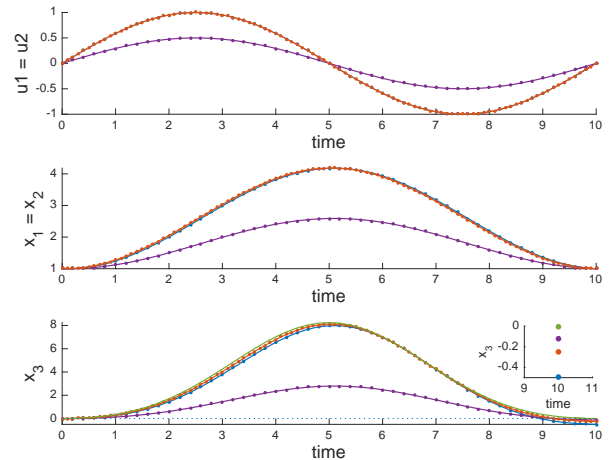

Fig. 3. Sinusoidal inputs for the discrete-time Brockett integrator (3). In blue the input has amplitude 1 and sampling time $h=0.2$ (the period is $T=10)$. In red the sampling time is $h=0.1$. In violet the amplitude of the input is reduced to 0.5 (and $h=0.2$ ). In green the integral curve of the continuous time system (1) is shown. The inset in the lower panel shows the end-point of the curve (i.e., the geometric phase accumulated by $x_{3}$ along a zero-area cycle).

where

$$
A_{1}=\left[\begin{array}{lll|l}
0 & 0 & 0 & 1 \\
0 & 0 & 0 & 0 \\
0 & 0 & 0 & 0 \\
\hline 0 & 0 & 0 & 0
\end{array}\right], \quad A_{2}=\left[\begin{array}{ccc|c}
0 & 0 & 0 & 0 \\
0 & 0 & 0 & 1 \\
1 & 0 & 0 & 0 \\
\hline 0 & 0 & 0 & 0
\end{array}\right] .
$$

When the piecewise constant input pattern

$$
u_{i}(t)= \begin{cases}0 & t<t_{1} \\ \alpha & t_{1} \leqslant t<t_{2} \\ 0 & t_{2} \leqslant t<t_{3}, \quad i=1,2 \\ -\alpha & t_{3} \leqslant t<t_{4} \\ 0 & t \geqslant t_{4}\end{cases}
$$

is applied to (10), the explicit solution one gets is

$$
\bar{x}\left(t_{2}\right)=e^{\left(A_{1}+A_{2}\right)\left(t_{2}-t_{1}\right) \alpha} \bar{x}(0)
$$

and

$$
\bar{x}\left(t_{4}\right)=e^{-\left(A_{1}+A_{2}\right)\left(t_{4}-t_{3}\right) \alpha} \bar{x}\left(t_{2}\right) .
$$

If $t_{2}-t_{1}=t_{4}-t_{3}$, then

$$
\bar{x}\left(t_{4}\right)=\bar{x}(0),
$$

as the two exponentials in (12) and (13) are one the inverse of the other. Hence indeed the geometric phase of (1) is zero for an input trajectory like (11).

In discrete-time, using homogeneous coordinates the system (3) becomes

$$
\bar{x}(k+1)=\left(I+h\left(A_{1} u_{1}+A_{2} u_{2}\right)\right) \bar{x}(k) .
$$

For the input pattern (4), this leads, at the end of the positive step to

$$
\bar{x}\left(k_{2}\right)=\left(I+h\left(A_{1}+A_{2}\right) \alpha\right)^{\ell} \bar{x}(0),
$$

and at the end of the negative step, to

$$
\bar{x}\left(k_{4}\right)=\left(I-h\left(A_{1}+A_{2}\right) \alpha\right)^{\ell} \bar{x}\left(k_{2}\right)
$$

(explicit expressions coincide obviously with (5)-(9)). However, the matrix $\left(I-\left(A_{1}+A_{2}\right) \alpha\right)^{\ell}$ is not the inverse of $\left(I+\left(A_{1}+A_{2}\right) \alpha\right)^{\ell}$. In particular

$$
\begin{array}{r}
\left(I-h\left(A_{1}+A_{2}\right) \alpha\right)^{\ell}\left(I+h\left(A_{1}+A_{2}\right) \alpha\right)^{\ell} \\
=\left[\begin{array}{ccc|c}
1 & 0 & 0 & 0 \\
0 & 1 & 0 & 0 \\
0 & 0 & 1 & -\ell h^{2} \alpha^{2} \\
\hline 0 & 0 & 0 & 0
\end{array}\right]
\end{array}
$$

meaning that the expression (9) is obtained for $x\left(k_{4}\right)$ at the end of the cycle.

For the system (1), instead of the Euler discretization (3), one can use an exact discretization. For that, it is enough to observe that the system (1) is nilpotent, which implies that the so-called Chen-Fliess series expansion corresponding to (1) can be computed exactly. It is however easier to work with matrix exponentials in the homogeneous coordinates. In fact, the matrices $A_{1}$ and $A_{2}$ are nilpotent: $A_{i}^{2}=0, i=1,2$, and the only nonzero matrix product is

$$
A_{2} A_{1}=\left[\begin{array}{ccc|c}
0 & 0 & 0 & 0 \\
0 & 0 & 0 & 0 \\
0 & 0 & 0 & 1 \\
\hline 0 & 0 & 0 & 0
\end{array}\right]
$$

Hence we have the exact series expansion

$$
\begin{aligned}
e^{\tau\left(A_{1} u_{1}+A_{2} u_{2}\right)} & =I+\tau\left(A_{1} u_{1}+A_{2} u_{2}\right)+\frac{\tau}{2 !}\left(A_{1} u_{1}+A_{2} u_{2}\right)^{2} \\
& =I+\tau\left(A_{1} u_{1}+A_{2} u_{2}\right)+\frac{\tau}{2 !} A_{2} A_{1} u_{1} u_{2} .
\end{aligned}
$$

Calling $h=\tau$, the system (1) admits the exact discretization given by the (complete) Taylor expansion

$$
\begin{gathered}
x(k+1)=x(k)+h\left[\begin{array}{c}
1 \\
0 \\
0
\end{array}\right] u_{1}(k)+h\left[\begin{array}{c}
0 \\
1 \\
x_{1}(k)
\end{array}\right] u_{2}(k) \\
+\frac{h^{2}}{2}\left[\begin{array}{c}
0 \\
0 \\
1
\end{array}\right] u_{1}(k) u_{2}(k) .
\end{gathered}
$$

The system (15) has no geometric phase when the input protocol (4) is applied. It is clear from (9) that the extra term distinguishing (15) from (3) is exactly equal to half the geometric phase at the end of a cycle induced by (4).

Remark 1 It is worth observing that when the input pattern of panels (a) and (b) of Fig. 2 is used, the Euler discretization (3) produces an exact result at the end of the cycle. In fact,

$$
u_{1}(k)=\left\{\begin{array}{ll}
0 & k<k_{1} \\
\alpha & k_{1} \leqslant k<k_{2} \\
0 & k_{2} \leqslant k<k_{3} \\
-\alpha & k_{3} \leqslant k<k_{4} \\
0 & k_{4} \leqslant k<k_{5} \\
0 & k \geqslant k_{5} .
\end{array}, \quad u_{2}(k)= \begin{cases}0 & k<k_{1} \\
0 & k_{1} \leqslant k<k_{2} \\
\alpha & k_{2} \leqslant k<k_{3} \\
0 & k_{3} \leqslant k<k_{4} \\
-\alpha & k_{4} \leqslant k<k_{5} \\
0 & k \geqslant k_{5}\end{cases}\right.
$$

implies that $u_{1}(k) u_{2}(k)=0 \forall k$, hence the extra term present in (15) always gives zero contribution. 


\section{GEOMETRIC PHASE AND DISCRETIZATION ERROR FOR DRIFTLESS NONLINEAR SYSTEMS}

Consider the continuous-time nonlinear driftless system linear in the inputs:

$$
\dot{x}=\sum_{i=1}^{m} g_{i}(x) u_{i}
$$

The Euler discretization is given by

$$
x(k+1)=x(k)+\sum_{i=1}^{m} g_{i}(x(k)) u_{i}(k) .
$$

For inputs $u_{i}$ that are piecewise-constant in each sampling interval $h$, an exact discretization of the system (16) is provided by a Taylor expansion [9]. It consists of the following infinite series

$$
x(k+1)=x(k)+\sum_{j=1}^{\infty} B^{[j]}(x(k), u(k)) \frac{h^{j}}{j !}
$$

where

$$
\begin{aligned}
B^{[1]}(x, u) & =\sum_{i=1}^{m} g_{i}(x) u_{i} \\
B^{[j+1]}(x, u) & =\frac{\partial B^{[j]}(x, u)}{\partial x} \sum_{i=1}^{m} g_{i}(x) u_{i} .
\end{aligned}
$$

The truncation error of the Euler discretization is then

$$
\epsilon(k)=\sum_{j=2}^{\infty} B^{[j]}(x(k), u(k)) \frac{h^{j}}{j !} .
$$

From the expressions (17) and (18), it is clear that $\lim _{h \rightarrow 0} \frac{x(k+h)-x(k)}{h}=\sum_{i=1}^{m} g_{i}(x(k)) u_{i}(k)$ and $\lim _{h \rightarrow \infty} \frac{\epsilon(k)}{h}=0$.

From the analysis carried out so far, the following somewhat obvious proposition gives the meaning of a geometric phase for discrete-time systems that are Euler discretizations.

Proposition 1 Consider the system (16) with $m=2$ and its Euler discretization (17). When the protocol (4) is applied to both inputs, the geometric phase produced by (17) is equal to the cumulant of the truncation error (20) corresponding to the input protocol (4).

Proof: Let us first show that, similarly to (1), also for (16) the continuous-time system with input protocol (11) for both inputs has zero geometric phase. Using formal exponentials, the flow of (16) under (11), $i=1,2$, is

$$
\begin{aligned}
& x\left(t_{2}\right)=e^{\left(t_{2}-t_{1}\right) \alpha \sum_{i=1}^{2} g_{i}} \circ x(0) \\
& x\left(t_{4}\right)=e^{-\left(t_{4}-t_{3}\right) \alpha \sum_{i=1}^{2} g_{i}} \circ x(2)
\end{aligned}
$$

Since the vector field in the two exponentials is the same, the Lie bracket is 0 and the Baker-Campbell-Hausdorff formula trivializes. Hence we can write

$$
x\left(t_{4}\right)=e^{\left(-\left(t_{4}-t_{3}\right) \sum_{i=1}^{2} g_{i}+\left(t_{2}-t_{1}\right) \sum_{i=1}^{2} g_{i}\right) \alpha} \circ x(0)=x(0)
$$

if $t_{4}-t_{3}=t_{2}-t_{1}$. By construction, the exact discretization overlaps with the continuous-time solution at the sampling instants, meaning that (18) has to have zero phase. Hence the geometric phase must correspond to the difference between (17) and (18), cumulated along the path followed.

Remark 2 The Proposition cannot be generalized to $m>2$, unless all inputs are subject to the same input protocol (4), or it is $\left[g_{i}(x), g_{j}(x)\right]=0$ whenever $u_{i} \neq u_{j}$. In fact from Remark 1, as soon as non-zero Lie brackets arise, the geometric phase is nonzero also for the exact Taylor discretization (since it is nonzero for the continuous-time system).

When the infinite series (19) can be computed in closed form, then it is possible to compare explicitly the solutions of the Euler and exact discretizations of a continuous-time system. Consider the following example

$$
\dot{x}=\left[\begin{array}{c}
1 \\
0 \\
\sin \left(x_{2}\right)
\end{array}\right] u_{1}+\left[\begin{array}{c}
0 \\
1 \\
\cos \left(x_{1}\right)
\end{array}\right] u_{2} .
$$

Its Euler discretization is

$x(k+1)=x(k)+h\left[\begin{array}{c}1 \\ 0 \\ \sin \left(x_{2}(k)\right)\end{array}\right] u_{1}(k)+h\left[\begin{array}{c}0 \\ 1 \\ \cos \left(x_{1}(k)\right)\end{array}\right] u_{2}(k)$

Computing the terms (19) explicitly, the exact Taylor discretization (18) is (the index $k$ in the right hand side is omitted to save some space):

$$
\begin{aligned}
& x(k+1)=x(k)+h\left[\begin{array}{c}
1 \\
0 \\
\sin \left(x_{2}\right)
\end{array}\right] u_{1}+h\left[\begin{array}{c}
0 \\
1 \\
\cos \left(x_{1}\right)
\end{array}\right] u_{2} \\
& +\left[\begin{array}{c}
0 \\
0 \\
\frac{\cos \left(x_{1}\right)}{u_{1}^{2}}\left(\sin \left(u_{1} h\right)-u_{1} h\right)+\frac{\sin \left(x_{1}\right)}{u_{1}^{2}}\left(\cos \left(u_{1} h\right)-1\right)
\end{array}\right] u_{1} u_{2} \\
& +\left[\begin{array}{c}
0 \\
0
\end{array}\right] u_{1} u_{2}
\end{aligned}
$$

The integral curves of the systems (22) and (23) are compared in Fig. 4 on the same zero-area shape trajectory of Fig. 2, panels (c) and (d). While (22) shows a geometric phase, (23) matches exactly (21) at all sampling instants.

\section{Discussion}

\section{A. System with/without drift.}

It is worth noticing that the restriction to driftless control systems is crucial, as continuous-time nonlinear systems with drift need not obey to the area rule, as can be verified on the following simple example

$$
\begin{aligned}
& \dot{x}_{1}=u_{1} \\
& \dot{x}_{2}=u_{2} \\
& \dot{x}_{3}=x_{1} x_{2} .
\end{aligned}
$$

If $x_{1}(0)=x_{2}(0)=0$ and $u_{1}=u_{2}$ periodic, this system forms zero-area cyclic shape space trajectories, but induces a nontrivial geometric phase on $x_{3}$. 


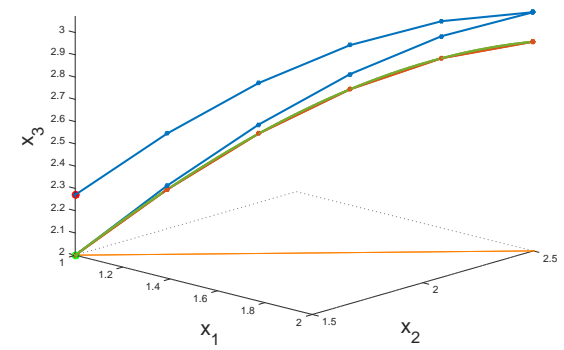

Fig. 4. Comparing Euler and exact discretization of (21). The Euler discretization (22) (in blue) shows a geometric phase. The exact discretization (23) (in red) shows no geometric phase and overlaps exactly with the true continuous-time system (green).

\section{B. "Drifting" with a single control input}

The geometric phase present in a discrete-time driftless system can be used in combination with periodic controls to induce a "stroboscopic" drift-like behavior in a system. For this it is enough to consider a single-input discrete-time system in $\mathbb{R}^{2}$, like (see [21], Lemma 4).

$$
\begin{aligned}
& x_{1}(k+1)=x_{1}(k)+u(k) \\
& x_{2}(k+1)=x_{2}(k)-r x_{1}(k) u(k) .
\end{aligned}
$$

Each time the input $u_{1}$ accomplishes a cyclic trajectory, a geometric phase is produced in $x_{2}$ while $x_{1}$ is unchanged. Such geometric phase accumulates when the cycle is repeated, see Fig. 5. In this specific example, similarly to (9), one gets that at the end of one cycle,

$$
x(k)=x(0)+\left[\begin{array}{c}
0 \\
r \ell
\end{array}\right] \alpha^{2} .
$$

If $k_{i+1}-k_{i}=\ell \forall i=1,2, \ldots$, and $p \in\left[k_{4 j}, k_{4 j+1}\right], j=$ $1,2, \ldots$, then the cumulation of such terms leads to

$$
x(p)=x(0)+j\left[\begin{array}{c}
0 \\
r \ell
\end{array}\right] \alpha^{2}
$$

see black dots in Fig. 5. This phenomenon is somewhat related to the "rectification of motion" of [6] (although the origin of the phase is different).

Notice that if the $u$ cycle is performed in the opposite order (i.e., first the negative step, then the positive step, see red curves in Fig. 5) the $x_{2}$ variable keeps "drifting" in the same direction due to the geometric phase. A consequence is that the discretization "drift" is not particularly useful for improving the controllability properties of a system. This is coherent with the conclusion of [21] that the system (25) is not controllable and neither "nearly-controllable".

\section{CONCLUSION}

In this paper we have shown through examples and explicit calculations that discrete-time nonlinear dynamical systems violate the so-called area rule for the geometric phase produced by cyclic motions in shape space. This violation is a sign that in discrete-time a "discretization phase" should be considered alongside the usual geometric phase of continuous-time systems.
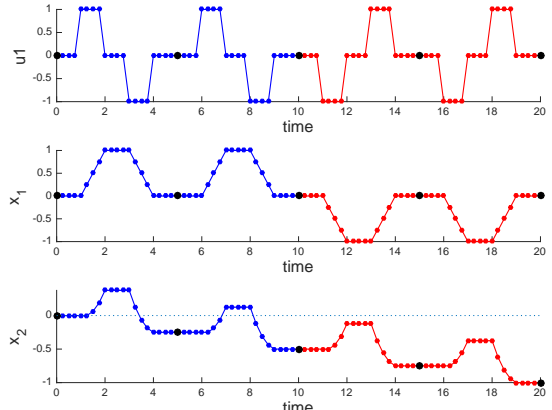

Fig. 5. Accumulation of the geometric phase yields a drift-like behavior For the system (25), the phase "drifts" in the same direction regardless of whether the input cycle consists of a step up followed by a step down (blue) or viceversa (red).

\section{REFERENCES}

[1] E. Aranda-Bricaire, Ü. Kotta, and C. H. Moog. Linearization of discrete-time systems. SIAM J. Contr. Optim., 34:1999-2023, 1996.

[2] M. Berry. Anticipations of the geometric phase. Physics Today, 43(12):34-40, 1990.

[3] P. Bettiol, B. Bonnard, L. Giraldi, P. Martinon, and J. Rouot. The Purcell Three-link swimmer: some geometric and numerical aspects related to periodic optimal controls. Preprint, 2015.

[4] A. M. Bloch. Nonholonomic Mechanics and Control, Springer, 2003.

[5] R. W. Brockett. Asymptotic stability and feedback stabilization. In Differential Geometric Control Theory, p. 181-91. Birkhauser, 1983.

[6] Roger W. Brockett. On the rectification of vibratory motion. Sensors and Actuators, 20:91-96, 1989.

[7] T. Chambrion, L. Giraldi, and A. Munnier. Optimal Strokes for Driftless Swimmers: A General Geometric Approach. Preprint, 2014.

[8] B. Jakubczyk, E. Sontag. Controllability of nonlinear discrete-time systems: a Lie-algebraic approach. SIAM J Contr. Optim., 28:1-33, 1990

[9] N. Kazantzis and C. Kravaris. Time-discretization of nonlinear control systems via taylor methods. Comp. \& Chem. Eng., 23:763 - 784, 1999.

[10] G. Lafferriere and H.H. Sussmann. A differential geometric approach to motion planning. In Z. Li and J.F. Canny, editors, Nonholonomic Motion Planning. Kluwer Academic, Boston, MA, 1993.

[11] J.P. Laumond. Nonholonomic motion planning versus controllability via the multibody car system example. Tech. Rep. STAN-CS-90-1345, Dep. of Computer Science, Stanford, 1990.

[12] J.P. Laumond, editor. Robot Motion Planning and Control. LNCIS Springer-Verlag, London, UK, 1998.

[13] J.E. Marsden and T.S. Ratiu. Introduction to Mechanics and Symmetry, Springer-Verlag, 2nd edition, 1999.

[14] C. Alden Mead. The geometric phase in molecular systems. Rev. Mod. Phys., 64:51-85, Jan 1992.

[15] S. Monaco and D. Normand-Cyrot. Nonlinear systems in discrete time. In M. Fliess and M. Hazewinkel, editors, Algebraic and Geometric Methods in Nonlinear Control Theory, pages 411-430. Springer Netherlands, Dordrecht, 1986.

[16] S. Monaco and D. Normand-Cyrot. Differential representations of driftless discrete-time dynamics. In Proceedings of the 37th CDC, $\mathrm{p}$. 4620-4625 vol.4, Dec 1998.

[17] S. Monaco and D. Normand-Cyrot. Issues on nonlinear digital control. European Journal of Control, 7(2?3):160 - 177, 2001.

[18] R. Montgomery. Gauge theory of the falling cat. In M.J. Enos, editor, Dynamics and control of mechanical systems: The falling cat and related problems, p. 193-218. AMS, Providence, RI, 1993.

[19] R. Murray and S. Sastry. Nonholonomic motion planning: steering with sinusoids. IEEE Trans. on Automatic Control, 38:700-716, 1993.

[20] R.M. Murray, Z. Li, S.S. Sastry, and S.S. Sastry. A Mathematical Introduction to Robotic Manipulation. Taylor \& Francis, 1994.

[21] L. Tie. On controllability of driftless inhomogeneous bilinear systems. In Proc. 53rd CDC, p. 3161-3166, Dec 2014.

[22] F. Wilczek and A. Shapere. Geometric Phases in Physics. World Sci., 1989. 